\title{
Process Dependencies and Process Interference Rules for Analyzing the Impact of Failure in a Service Composition Environment
}

\author{
Yang Xiao and Susan D. Urban \\ Department of Computer Science and Engineering \\ Arizona State University \\ PO Box 878809 Tempe, AZ, 85287-8809 USA \\ \{yang.xiao, s.urban\} @asu.edu
}

\begin{abstract}
This paper presents a process dependency model for dynamically analyzing data dependencies among concurrently executing processes in an autonomous, distributed service composition environment. Data dependencies are derived from incremental data changes captured at each service execution site. Deltas are then used within a rule-based recovery model to specify how failure recovery of one process can potentially affect another process execution based on application semantics. This research supports relaxed isolation and application-dependent semantic correctness for concurrent process execution, with a unique approach to resolving the impact of process failure recovery on other processes, using data dependencies derived from distributed, autonomous services.
\end{abstract}

Keywords: Data Dependencies, Process Interference, Service Composition.

\section{Introduction}

Web Services and Grid Services have become widely used for B2B integration. However, the loosely-coupled, autonomous nature of services poses new challenges for the correctness of concurrent execution of global processes that are composed of distributed services. Most processes that are composed of Web Services must execute using a relaxed notion of isolation since individual service invocations can unilaterally commit before a process completes [15]. Relaxed isolation leads to dirty reads and writes and also calls for a more user-defined approach to the correctness of concurrent execution. Previous work with advanced transaction models [4] and transactional workflows [19] has used compensation to semantically undo a process. However, existing research has not fully addressed the process interference [20] that is introduced when the recovery of one process affects other concurrently-executing processes due to data changes introduced by the compensation of a failed process. A robust service composition environment should not only recover a failed process, but should also make sure process interference is properly handled based on application semantics.

This research has defined a process dependency model to dynamically analyze the data dependencies among concurrently executing processes that are composed of 
distributed services, providing a rule-based approach to resolve process interference based on application-dependent semantic correctness. The research has been conducted in the context of the DeltaGrid project, where services known as DeltaEnabled Grid Services (DEGS) [2] are extended with the capability of recording incremental data changes, known as deltas. Deltas from distributed service execution sites are merged to create a global, time-ordered schedule of delta values within a Process History Capture System (PHCS) [20, 21]. The merged schedule of deltas then provides a means for tracking data dependencies among concurrently executing processes to determine how the failure of one process can potentially affect other processes. Once the potential process interference is identified, deltas can also be queried using process interference rules $(P I R)$ to apply user-defined semantic correctness conditions that determine whether an affected process should keep running or invoke its own recovery procedures.

Based on the service composition model presented in [20, 22], the focus of this paper is on the specification of the process dependency model, the definition of process interference rules, and the illustration of the way in which process interference rules can be used to query deltas to impose user-defined correctness conditions as part of the recovery process [20]. This research contributes towards ensuring a semantically robust, concurrent process execution environment for distributed, autonomous service composition, by dynamically analyzing data dependencies among concurrently executing processes and by providing a rule-based approach to resolve process interference based on application semantics.

The rest of this paper is organized as follows. After outlining related work in Section 2, the paper provides an overview of the DeltaGrid abstract execution model in Section 3. Section 4 presents the process dependency model, while Section 5 elaborates on the use of process interference rules. The paper concludes in Section 6 with a summary of our implementation of the global execution history and process interference rules as well as a discussion of future research.

\section{Related Work}

Research on exception handling in a service composition environment has primarily focused on implementing ACID transaction semantics. Open nested transactions over Web Services are supported in [12], contingency is applied to forward recover a composite service in [16], and WS-Transaction [3] defines processes as either Atomic Transactions with ACID properties or Business Activities with compensation capabilities. An agent-based transaction model (ABT) [7] integrates agent technologies in coordinating Web Services to form a transaction. To avoid the cost of compensation, tentative holding is used in [9] to achieve a tentative commit state for transactions over Web Services. Acceptable Termination States (ATS) [1] are used to ensure userdefined failure atomicity of composite services. Active rules have been used to handle service exceptions independent of application logic, such as service availability, selection, and enactment [14, 23], or search for substitute services when an application exception occurs [10]. But the question of how the recovery of a composite process could possibly affect other concurrently executing processes has not been addressed. 
Advanced transaction models support relaxed isolation for long running transactions composed of subtransactions. Sagas [6] can be backward recovered by compensating each task in reverse order. The flexible transaction model [23] executes an alternative path when the original path fails. The backward recovery of a failed transaction causes cascaded rollback or compensation of other transactions that are read or write dependent on the failed transaction. Instead of cascaded rollback, only transactions dependent on tainted data produced by a flawed transaction are removed in [11]. However this work is conducted in a centralized database system which relies on an extended database engine to capture read dependency information.

Research in the transactional workflow area has adopted compensation as a backward recovery technique $[5,8,18]$ and explored the handling of data dependencies among workflows [8, 18]. The ConTract model [18] uses pre-conditions integrated into a workflow script to determine whether a compensation of a step affects another step execution. However, read dependency is not considered. WAMO [5] defines a flexible recovery framework for a workflow without considering process interference. In CREW [8], a static specification on the equivalence of data items across workflows is required to track data dependencies.

The research presented in this paper supports relaxed isolation and applicationdependent semantic correctness for concurrent process execution, with a rule-based approach to resolving the impact of process failure and recovery on other concurrently executing processes [20]. Instead of statically specifying data dependencies, this research dynamically analyzes write dependencies and potential read dependencies among concurrently executing processes by capturing and merging data changes from distributed service execution, providing an intelligent approach to discovering dependencies among processes in an autonomous service composition environment and using data dependencies to support failure recovery.

\section{Overview of the DeltaGrid Abstract Execution Model}

Our research has developed the DeltaGrid system as a semantically robust execution environment for distributed processes executing over Delta-Enabled Grid Services (DEGS). A DEGS is a Grid Service that has been enhanced with an interface for accessing the deltas that are associated with service execution [2]. The work in [2] has demonstrated the manner in which incremental data changes can be captured from data-centric services, using features such as triggers as well as facilities for monitoring and externalizing database log files, such as Oracle Streams. Deltas collected from DEGSs are forwarded to a Process History Capture System (PHCS) [21] to form a global execution history where data dependencies among concurrently executing processes can be dynamically analyzed. The merged deltas from distributed sites also form the basis for analyzing process interference, determining the effect that the failure recovery of one process can have on other concurrently executing processes using application semantics.

In support of our research, we have defined an abstract execution model for studying the manner in which data changes from DEGS can be used to analyze the dependencies that exist among concurrent processes. Fig. 1 shows the DeltaGrid abstract execution model, which is composed of three components: the service composition and recovery 


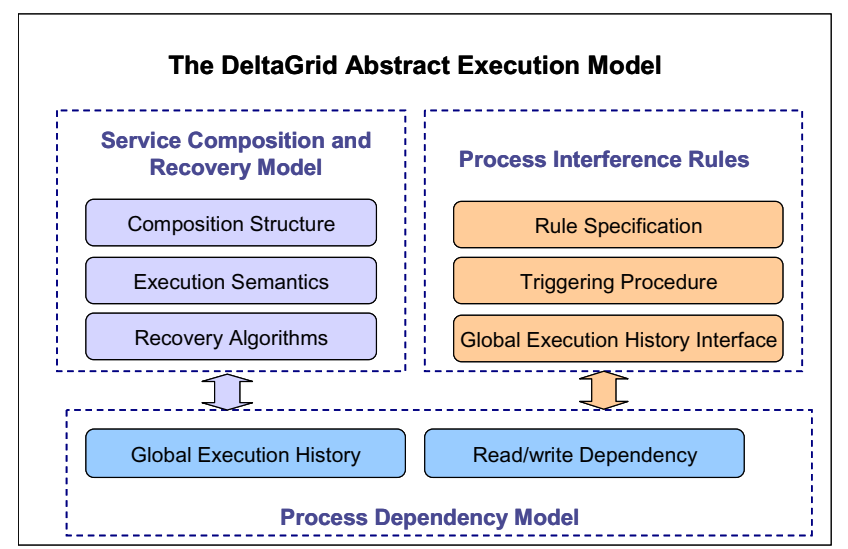

Fig. 1. The DeltaGrid abstract execution model

model, the process dependency model, and process interference rules. The service composition model defines a hierarchical service composition structure as well as the semantics of execution entities for the handling of operation execution failure occurring at any compositional level. The recovery model resolves a service execution failure within a process. Recovery techniques, such as compensation for logical backward recovery, contingency for forward recovery, and a process known as delta-enabled rollback for physical rollback of a completed service, are applied at different composition levels to maximize the recovery of a failed process. The details of the composition model and recovery model can be found in [20,22].

The process dependency model further defines the relationships that exist among concurrently executing processes in terms of write dependencies and potential read dependencies. The process dependency model also defines how data dependencies can be dynamically analyzed from a global process execution history. Process interference rules are active rules that query the data changes from a failed process and its read and write dependent processes, using application semantics to determine if the recovery of a failed process has an affect on active processes that are read and/or write dependent on the failed process. The rest of this paper presents the process dependency model and illustrates the use process interference rules in the recovery process.

\section{The Process Dependency Model}

As described in the previous section, deltas from distributed service executions are forwarded to a Process History Capture System. These deltas are merged to form a time-ordered sequence of data changes that are used to analyze data dependencies [21]. This section presents the process dependency model. Section 4.1 defines the global execution history, integrating process execution context and deltas from distributed sites. Section 4.2 defines write dependency and how to derive write dependencies from deltas. Section 4.3 defines potential read dependency and how to 
analyze potential read dependency from the process execution context. Section 4.4 then provides a case study to illustrate the use of the process dependency model to discover write dependencies among concurrently executing processes.

\subsection{Global Execution History}

The global execution history of concurrently executing, distributed processes is composed of 1) the execution context of each process $\mathrm{p}_{\mathrm{i}}$, and 2) the merged deltas

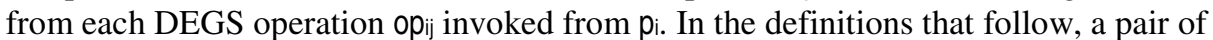
square brackets [] indicates a partially ordered list of elements ordered by the timestamp associated with each delta. Each op $\mathrm{ij}$ is also associated with one DEGS through a degsID.

Definition 1 (Delta): A delta represents an incremental value change on an attribute of an object generated by execution of a DEGS operation. A delta is a six-element tuple, denoted as $\Delta\left(\mathrm{olD}, \mathrm{a}, \mathrm{V}_{\text {old }}, \mathrm{V}_{\text {new }}, \mathrm{t} \mathrm{s}_{\mathrm{n}}, \mathrm{op}_{\mathrm{ii}}\right)$, representing an object's attribute value change produced by an operation at a specific time. A delta contains an object identifier (olD) indicating the changed object, an attribute name (a) indicating the changed attribute, the old value of the attribute $\left(V_{\text {old }}\right)$ before the execution of the operation, the new value of the attribute $\left(V_{\text {new }}\right)$ created by the operation, a timestamp $\left(t_{n}\right)$ indicating the time of the new attribute value that is generated, and the identifier of the operation $\left(\mathrm{op}_{\mathrm{ij}}\right)$ that has created this delta.

As an autonomous entity, a DEGS produces a local execution history.

Definition 2 (DEGS Local Execution History): A DEGS local execution history $\mathrm{ln}($ degs $I D)$ is a three-element tuple, denoted as $\operatorname{lh}($ degs $\mid D)=\left\langle t \mathrm{~s}_{\mathrm{s}}, \mathrm{ts}_{\mathrm{e}}, \delta(\right.$ degs $\left.\mid \mathrm{D})\right\rangle$, where:

-tss and tse are the start time and end time of a DEGS execution history, respectively.

$-\delta$ (degs $I D)$ is a time-ordered sequence of deltas that are generated by operations that are executed at a specific DEGS during the time frame formed by $t_{s}$ and tse, denoted

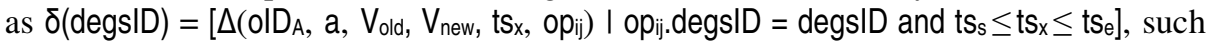
that the order of the list is based on the delta creation time ts $\mathrm{x}$.

A DEGS operation execution creates an execution context for an operation.

Definition 3 (Operation Execution Context): An operation execution context is a five-element tuple, denoted as ec $\left(\mathrm{op}_{\mathrm{ij}}\right)=\left\langle\mathrm{ts}_{\mathrm{s}}\right.$, $\left.t \mathrm{~s}_{\mathrm{e}}, \mathrm{I}, \mathrm{O}, \mathrm{S}\right\rangle$. The operation execution

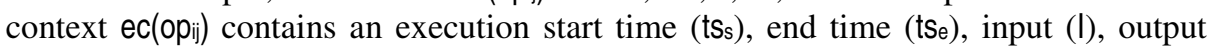
$(\mathrm{O})$, and an execution state (S) as defined in the operation execution semantics in the service composition model [20,22].

As the enclosing entity of an operation, a process also has its own execution context $\mathrm{ec}\left(\mathrm{p}_{\mathrm{i}}\right)=\langle\mathrm{ts}$ s, tse, I, O, S $\rangle$ that corresponds to the definition of the operation execution context, but defines the context at the process level.

Each DEGS local execution history together with the process execution context of each process forms the global execution context.

Definition 4 (Global Execution Context): A global execution context is a timeordered sequence of runtime context information for operations and processes that 
occur within a certain time frame formed by $t s_{s}$ and $t s_{e}\left(t s_{s}<t s_{e}\right)$. The global execution context is denoted as gec $=\left[\right.$ ec(entity) I (entity $=0 p_{i j}$ or entity $\left.=p_{i}\right)$ and $\left(t_{s} \leq e c(e n t i t y) . t s_{s}<\right.$ $\left.\mathrm{ec}(\mathrm{entity}) . t \mathrm{~s}_{\mathrm{e}} \leq \mathrm{ts} \mathrm{s}_{\mathrm{e}}\right)$ ], where entity represents either an operation $0 \mathrm{p}_{\mathrm{ij}}$ or a process $\mathrm{p}_{\mathrm{i}}$.

Integrating local execution histories of DEGSs and the global execution context, the global execution history provides the basis to analyze write dependencies among concurrently executing processes by the provision of a time-ordered object access schedule.

Definition 5 (Global Execution History): A global execution history is an integration of individual DEGS execution histories within the time frame formed by start time $t s_{s}$ and end time $t s_{e}$, denoted as $g h=\left\langle t s_{s}\right.$, $\left.t s_{e}, \delta g, g e c\right\rangle$, where $\delta g$ is a timeordered sequence of deltas that are generated by distributed operation execution, denoted as $\delta g=\left[\Delta\left(o_{\mid l} D_{A}, a, V_{\text {old }}, V_{\text {new }}, t s_{x}, o p_{i j}\right) \mid t s_{s} \leq t s_{x} \leq t s_{e}\right]$, and gec is the global execution context within the given time frame.

Definition 6 (System Invocation Event Sequence): A system invocation event sequence is an ordered list of events that are associated with the invocation of an execution entity based on the event occurrence timestamp. A system invocation event is denoted as entity, such that e indicates an invocation event and entity indicates an execution entity as the event source. System events include the invocation of a process $\left(e_{\mathrm{pi}}\right)$, an operation $\left(\mathrm{e}_{\mathrm{opij}}\right)$, or a failure recovery event such as compensation of an operation $\left(e_{\text {copij }}\right)$. A system invocation event sequence is denoted as $E_{\text {seq }}=\left[e_{\text {entity }} \mid\right.$ entity $=$ op $_{\mathrm{ij}}$ or entity $=\mathrm{p}_{\mathrm{i}}$.

Fig. 2 illustrates a process execution scenario with two concurrently executing processes $p_{1}$ and $p_{2}$ shown at the top of the Figure. The process $p_{1}$ is composed of operations $o p_{11}, o p_{12}, o p_{13}$ and $o p_{14}$. The process $p_{2}$ contains $o p_{21}$ and $o p_{22}$. These operations execute on two different sites DEGS 1 and DEGS 2 . The operations $\mathrm{op}_{11}, \mathrm{op}_{21}$, $\mathrm{op}_{13}$ and $\mathrm{op}_{14}$ are provided by $\mathrm{DEGS} \mathrm{S}_{1}$, while $\mathrm{op}_{12}$ and $\mathrm{op}_{22}$ are provided by $\mathrm{DEGS}$. The horizontal coordinate indicates the time frame starting from ts to tse.

The bottom part of the diagram shows the deltas generated by each operation, ordered by the timestamp of each delta. DEGS ${ }_{1}$ contains deltas for objects $X$ and $Y$, and $D E G S_{2}$ contains deltas for object $Z$. Each item represents a delta for a specific attribute of an object. For example, $X_{2}$ indicates a delta for object $X$ where the subscript identifies the sequence of the delta with respect to the creation time. The process execution scenario illustrates:

- DEGS's local execution history $\ln \left(D \mathrm{DGS}_{1}\right)$ contains all of the deltas that are created by operations that execute on $D E G S_{1}$, denoted as $\ln \left(D E G S_{1}\right)=<t s_{s}$, tse,

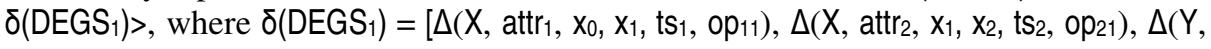
attr $\left.\left._{1}, \mathrm{y}_{0}, \mathrm{y}_{1}, \mathrm{ts}_{2}, \mathrm{op}_{21}\right), \Delta\left(\mathrm{X}, \operatorname{attr}_{1}, \mathrm{x}_{2}, \mathrm{x}_{3}, \mathrm{ts}_{4}, \mathrm{op}_{13}\right), \Delta\left(\mathrm{X}, \mathrm{attr}_{1}, \mathrm{x}_{3}, \mathrm{X}_{4}, \mathrm{ts}_{6}, \mathrm{op}_{14}\right)\right]$.

- DEGS2's local execution history $\ln \left(\mathrm{DEGS}_{2}\right)=\left\langle\mathrm{ts}_{\mathrm{s}}\right.$, tse, $\delta\left(\mathrm{DEGS}_{2}\right)>$, where $\delta\left(\mathrm{DEGS}_{2}\right)$ $=\left[\Delta\left(\mathrm{Z}, \operatorname{attr}_{1}, \mathrm{Z}_{0}, \mathrm{Z}_{1}, \mathrm{ts}_{3}, \mathrm{op}_{12}\right), \Delta\left(\mathrm{Z}, \mathrm{attr}_{2}, \mathrm{Z}_{1}, \mathrm{Z}_{2}, \mathrm{ts}_{5}, \mathrm{op}_{22}\right)\right]$.

- The global execution history $\mathrm{gh}=\left\langle\mathrm{ts}_{\mathrm{s}}\right.$, tse, $\delta \mathrm{g}$, gec $>$, where $\delta \mathrm{g}$ integrates $\delta\left(D E G S_{1}\right)$ and $\delta\left(D E G S_{2}\right)$, ordering each delta in time order. $\delta g=\left[\Delta\left(X\right.\right.$, attr $\left.1, x_{0}, x_{1}, t_{1}, o_{11}\right), \Delta(X$, $\left.\operatorname{attr}_{2}, \mathrm{X}_{1}, \mathrm{X}_{2}, \mathrm{ts}_{2}, \mathrm{op}_{21}\right), \Delta\left(\mathrm{Y}, \mathrm{attr}_{1}, \mathrm{y}_{0}, \mathrm{y}_{1}, \mathrm{ts}_{2}, \mathrm{op}_{21}\right), \Delta\left(\mathrm{Z}, \mathrm{attr}_{1}, \mathrm{z}_{0}, \mathrm{Z}_{1}, \mathrm{ts}_{3}, \mathrm{op}_{12}\right), \Delta\left(\mathrm{X}, \mathrm{attr}_{1}, \mathrm{x}_{2}, \mathrm{x}_{3}\right.$, $\left.\left.\mathrm{ts}_{4}, \mathrm{op}_{13}\right), \Delta\left(\mathrm{Z}, \mathrm{attr}_{2}, \mathrm{Z}_{1}, \mathrm{Z}_{2}, \mathrm{ts}_{5}, \mathrm{op}_{22}\right), \Delta\left(\mathrm{X}, \operatorname{attr}_{1}, \mathrm{x}_{3}, \mathrm{x}_{4}, \mathrm{ts}_{6}, \mathrm{op}_{14}\right)\right]$.

- The system invocation event sequence indicates the invocation of each operation, ordered by operation start time. $E_{\text {seq }}=\left[e_{o p 11}, e_{o p 21}, e_{o p 12}, e_{o p 13}, e_{o p 22}, e_{o p 14}\right]$. 


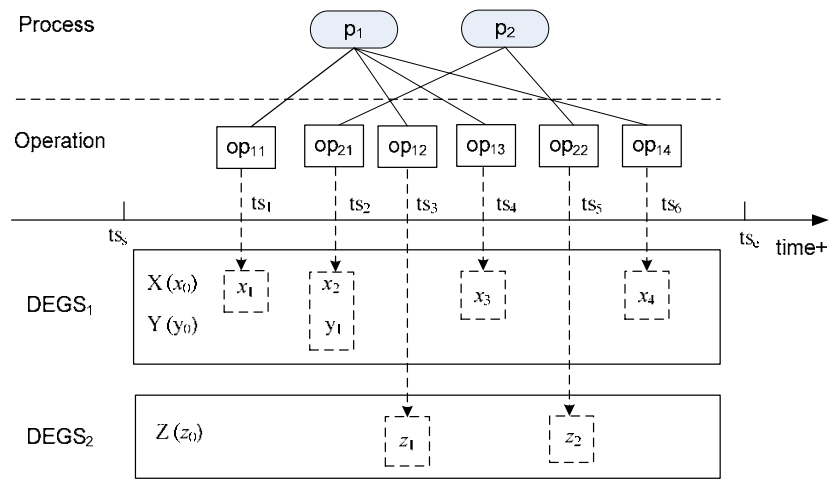

Fig. 2. A process execution scenario

\subsection{Write Dependency}

The merged deltas $\delta g$ of the global execution history can be used to identify the active processes that are dependent on a failed process.

Definition 7 (Process-Level Write Dependency): A process-level write dependency exists if a process $p_{i}$ writes an object $x$ that has been written by another process $p_{j}$ before $p_{j}$ completes $(i \neq j)$. In this case, $p_{i}$ is write dependent on $p_{j}$ with respect to $x$, denoted as $p_{i} \rightarrow{ }_{w} p_{j}$.

Definition 8 (Operation-Level Write Dependency): An operation-level write dependency exists if an operation op ik of process $p_{i}$ writes an object that has been written by another operation $0 \mathrm{p}_{\mathrm{jl}}$ of process $\mathrm{p}_{\mathrm{j}}$, denoted as $\mathrm{o}_{\mathrm{ik}} \rightarrow{ }_{\mathrm{w}} \mathrm{o} \mathrm{p}_{\mathrm{j}}$. Operation-level write dependency can exist between two operations within the same process $(i=j)$.

The operations that are write dependent on a specific operation $\mathrm{op}_{\mathrm{jl}}$ form $o \mathrm{p}_{\mathrm{j}}$ 's write dependent set.

Definition 9 (Operation-Level Write Dependent Set for an Operation): An operation opj's operational-level write dependent set is the set of all operations that are write dependent on $\mathrm{Op}_{\mathrm{j}}$, denoted as $\mathrm{wdop}_{\mathrm{op}}\left(\mathrm{Op}_{\mathrm{j}} \mathrm{I}\right)=\left\{\mathrm{op}_{\mathrm{ik}} \mid \mathrm{op}_{\mathrm{ik}} \rightarrow \mathrm{w}_{\mathrm{w}} \mathrm{op}\right\}$.

If opik is write dependent on $\mathrm{op}_{\mathrm{j}}\left(\mathrm{O} \mathrm{p}_{\mathrm{ik}} \rightarrow \mathrm{w}_{\mathrm{w}} \mathrm{O}(\mathrm{i} \neq \mathrm{j})\right)$, the enclosing process of opikis also write dependent on $\mathrm{Op} \mathrm{p}_{\mathrm{j}}\left(\mathrm{p}_{\mathrm{i}} \rightarrow \mathrm{w} \mathrm{Op}_{\mathrm{j}}\right)$.

Operation-level write dependencies can be derived from the global execution history gh. Assume two operations opik and opj il have modified the same object olDA. In gh, we observe $\delta g=\left[\ldots, \Delta\left(\right.\right.$ olD $\left._{A}, a, V_{\text {old }}, V_{\text {new }}, t_{\mathrm{s} x}, \mathrm{op}_{\mathrm{j}}\right), \ldots, \Delta\left(\mathrm{ol} \mathrm{D}_{\mathrm{A}}, \mathrm{b}, \mathrm{V}_{\text {old }}, \mathrm{V}_{\text {new, }}, \mathrm{tsy}_{\mathrm{y}}, \mathrm{op}\right.$ ik $\left.), \ldots\right]$, where $t \mathrm{~s}_{\mathrm{x}}<t \mathrm{ts}_{\mathrm{y}}$. Then $\delta \mathrm{g}$ indicates that at the operation level, op $\mathrm{ik} \in \mathrm{wdop}_{\mathrm{op}}\left(\mathrm{op}_{\mathrm{j}}\right)$.

\subsection{Potential Read Dependency}

Since a DEGS does not capture read information, the global execution context can be used to reveal potential read dependency among operations. An operation $o p_{i k}$ is potentially read dependent on another operation opj under the following conditions: 
1) $o p_{i k}$ and $o p_{j l}$ execute on the same DEGS, denoted as ec(op $\left(o p_{i k}\right) \cdot \operatorname{degs} I D=e c\left(o p_{j l}\right)$.degs ID. 2) the execution duration of $o p_{i k}$ and $o p_{j l}$ overlaps, or $o p_{i k}$ is invoked after the termination of opjl.

Fig. 3 shows various execution duration overlap scenarios of op $i$ and $o p_{j l}$ In (a) and

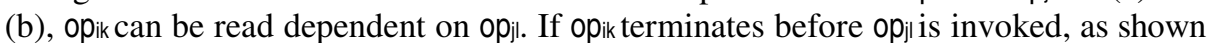
in (c), it is not possible for $\mathrm{op}_{\mathrm{ik}}$ to be read dependent on $\mathrm{op}_{\mathrm{j} \text {. }}$

The read dependency conditions in (a) and (b) can be expressed using operation

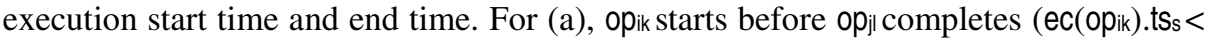
ec $\left.\left(o p_{j l}\right) . t s_{e}\right)$, and ends after op $p_{j l}$ starts $\left(e c\left(o p_{i k}\right) . t s_{e}>\operatorname{ec}\left(o p_{j l}\right) . t s_{s}\right)$. For $(b)$, op ik must start after $o p_{j l}$ completes $\left(e c\left(o p_{i k}\right) . t s_{s}>e c\left(o p_{j l}\right) . t s_{e}\right)$. Thus op $p_{i k}$ is potentially read dependent on

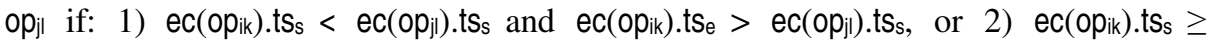
ec $\left(\mathrm{op}_{\mathrm{j}}\right) . \mathrm{ts}$.

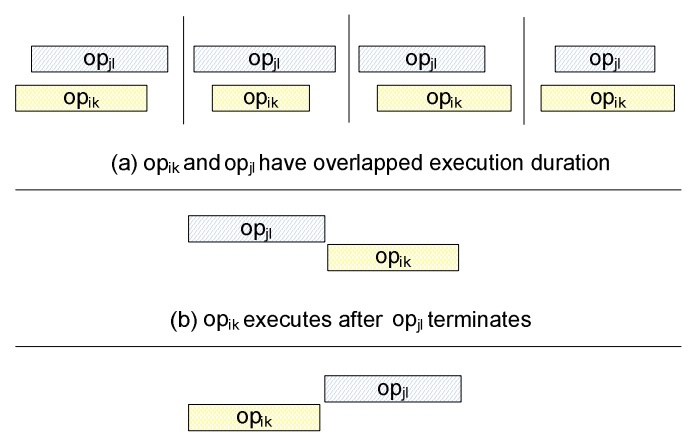

(c) $\mathrm{op}_{\mathrm{ik}}$ terminates before op $\mathrm{p}_{\mathrm{j}}$ executes

Fig. 3. Execution scenarios of op $\mathrm{p}_{\mathrm{ik}}$ and $\mathrm{op}_{\mathrm{j} \mathrm{l}}$

Potential read dependency can be defined at the process or operation levels.

Definition 10 (Process-Level Read Dependency): A process-level read dependency exists if a process $p_{i}$ reads an object $x$ that has been written by another process $p_{j}$ before $p_{j}$ completes $(i \neq j)$. In this case, $p_{i}$ is read dependent on $p_{j}$ with respect to $x$, denoted as $p_{i} \rightarrow r p_{j}$.

Definition 11 (Operation-Level Read Dependency): An operation-level read dependency exists if an operation $o p_{i k}$ of process $p_{i}$ reads an object that has been written by another operation $0 p_{j l}$ of process $p_{j}$, denoted as $o p_{i k} \rightarrow{ }_{r} \mathrm{op}_{j l}(i \neq j)$.

The operations that are potentially read dependent on an operation $0 p_{j l}$ form a set referred to as op $\mathrm{p}_{\mathrm{j}}$ 's read dependent set.

Definition 12 (Operation-Level Read Dependent Set for an Operation): An operation $o p_{j l}$ 's operational-level read dependent set is a set of all operations that are

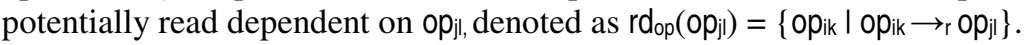

If $\mathrm{op}_{\mathrm{ik}}$ is read dependent on $\mathrm{op}_{\mathrm{jl}}\left(\mathrm{op}_{\mathrm{ik}} \rightarrow \mathrm{rop}(\mathrm{i} \neq \mathrm{j})\right)$, the enclosing process of op $\mathrm{p}_{\mathrm{ik}}$ is also read dependent on $\mathrm{op}_{\mathrm{jl}}\left(\mathrm{p}_{\mathrm{i}} \rightarrow \mathrm{r} \mathrm{op}_{\mathrm{j}}\right)$.

Assume an operation $0 \mathrm{p}_{\mathrm{ij}}$ is compensated and the evaluation of process interference requires the identification of processes that are potentially read dependent on $\mathrm{op}_{\mathrm{ij}}$. 
Suppose in the global execution context, we observe the system invocation event sequence $E_{s e q}=\left[\ldots, e_{o p i j}, e_{o p k m}, e_{o p x y}, e_{c o p i j}\right]$. $E_{s e q}$ shows that $o_{k m}$ and $o p_{x y}$ are invoked after $o p_{i j} s t a r t s\left(e c\left(o p_{k m}\right) . t s_{s} \geq e c\left(o p_{i j}\right) \cdot t s_{s}\right)$. If $o p_{k m}$ and $o p_{i j}$ execute on the same DEGS $\left(e c\left(o p_{k m}\right) \cdot \operatorname{degs} I D=e c\left(o p_{i j}\right) \cdot \operatorname{degsID}\right)$, then $o p_{k m} \rightarrow o_{i j}$. Thus the enclosing process $p_{k}$ of $o p_{k m}$ is potentially read dependent on $\mathrm{op}_{\mathrm{ij}}$.

\subsection{Case Study}

An online shopping case study has been used to illustrate the tracking of write dependencies among concurrent processes. The case study will be used again in Section 5 to illustrate the use of process interference rules.

The online shopping application contains typical business processes that describe the activities conducted by shoppers, the store, and vendors. For example, the process placeClientOrder is responsible for invoking services that place client orders and decrease the inventory quantity. The process replenishlnventory invokes services that increase the inventory quantity when vendor orders are received.

Fig. 4 shows write dependency between two instances of the process placeClientOrder $\left(p_{c 1}\right.$ and $p_{c 2}$ ) and an instance of the process replenishlnventory $\left(p_{r}\right)$. The top part of the diagram shows the executed operations in time sequence order. The bottom part shows deltas generated by an operation's execution. To keep the case simple, we use three objects (I from DEGS $1, C A$ and CB from DEGS ${ }_{2}$ ) to demonstrate the existence of write dependency. All three process instances are related with the same inventory item identified by object I. Process instances $p_{\mathrm{c} 1}$ and $p_{\mathrm{c} 2}$ have created two different orders, identified as $\mathrm{CA}$ and $\mathrm{CB}$.

The processes $p_{c 1}, p_{c 2}$, and $p_{r}$ start at different times. Process $p_{c 1}$ 's current operation is packOrder and $p_{c 2}$ 's current operation is declnventory. Process $p_{r}$ is in the process of backward recovery since the items that have entered the inventory by operation inclnventory are recalled by the vendor due to quality problems. Process pr's recovery procedure contains compensating operations packBackOrder, declnventory and inclnventory.

The global execution history for this scenario shows that write dependency exists among $p_{c 1}, p_{c 2}$, and $p_{r}$. The process $p_{c 2}$ is write dependent on $p_{c 1}$ and $p_{r}$. The process $p_{r}$ is write dependent on $p_{c 1}$ and $p_{c 2}$. The process $p_{c 1}$ is not write dependent on $p_{c 2}$ or $p_{r}$. The operation $p_{\mathrm{c} 2}$.declnventory decreases the quantity of the inventory item I that has been increased by $p_{r}$.declnventory. Then $p_{r}$.declnventory's compensation $p_{r}$.cop:declnventory modified the value of I by decreasing the value. This modification could potentially affect the execution of $p_{c 2}$ if the number of required inventory items for the client order is no longer available. If the recovery procedure of $p_{r}$ does affect $p_{c 2}$ 's execution (i.e., process interference exists between $p_{r}$ and $p_{c 2}$ ), $p_{c 2}$ needs to be recovered. Otherwise $p_{\mathrm{c} 2}$ can keep running. Whether a cascading recovery is necessary is determined by process interference rules defined in Section 5.

Space does not permit the presentation of a full example of identifying read dependencies. It is important to remember that read dependencies are not derived from the schedule of delta values but are instead derived from the execution context, which identifies the overlapping execution timeframe of concurrent processes on the same service. As such, we refer to read dependencies as potential read dependencies since information about the specific data items that have been read is not available. 


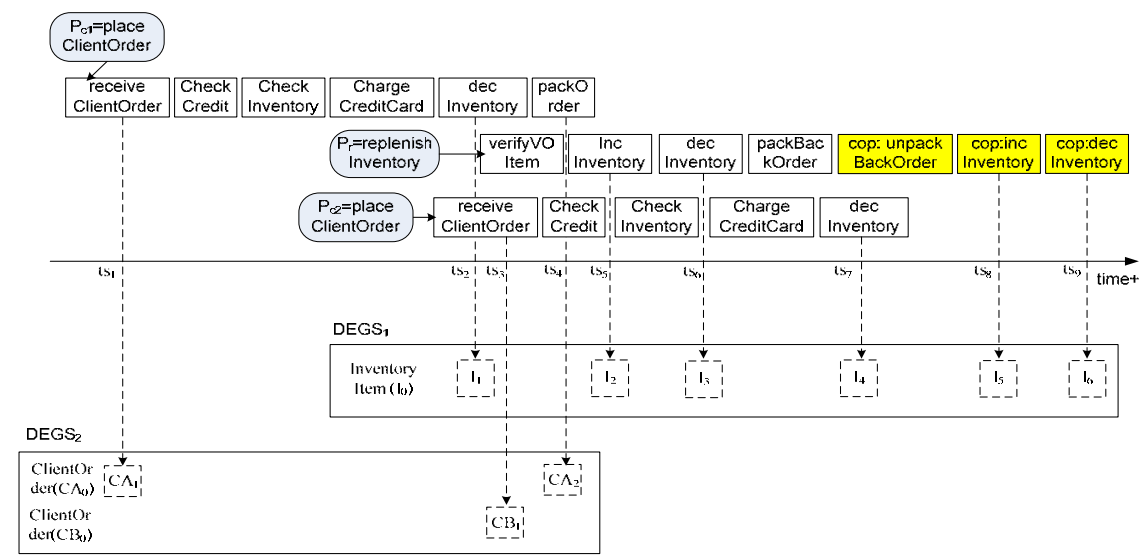

Fig. 4. Write dependency among multiple processes

Process interference rules as described in the next section can be used to determine if the failed process has accessed and modified any of the data items that are critical to the execution of the potentially read dependent process.

\section{Process Interference Rules}

The previous section illustrated the analysis of write dependencies and potential read dependencies from the global execution history. In a typical environment that supports relaxed isolation, the failure of one process causes cascaded recovery of other dependent processes. In the DeltaGrid environment, we use process interference rules (PIRs) to determine if the cascaded recovery of a dependent process is necessary. Process interference rules query the delta values of the global execution history, using user-defined application semantics to determine if an affected process should keep running or invoke its own recovery procedures. Section 5.1 defines the structure of a PIR and the way in which it queries the global execution history object model. Section 5.2 then presents a PIR example.

\subsection{PIR Definition}

A PIR is written from the perspective of an executing process $\left(p_{e}\right)$ that is interrupted by the recovery of an unknown failed process $\left(\mathrm{p}_{\mathrm{f}}\right)$. The interruption occurs because $\mathrm{p}_{\mathrm{e}}$ is identified as being write dependent or potentially read dependent on pf. PIRs are expressed using an extended version of the Integration Rule Language (IRL) [13, 17], which was originally defined to provide a rule-based approach to component integration.

A process interference rule has four elements: event, define, condition, and action, as shown in Fig. 5. The triggering event of a PIR is a write dependent or a read dependent event. A write dependent event is triggered after the backward recovery of a failed process (failedProcess) if the failedProcess has at least one write dependent process. The format of a 
write dependent event is: <writeDependentProcessName>WriteDependency (failedProcess, wdProcess). The write dependent event contains the name of the write dependent process instance (writeDependentProcessName), and two parameters: the identifier of the failed process (failedProcess) and the identifier of the write dependent process instance (wdProcess). In Fig. 4, after the compensation of the failed process replenishlnventory $\left(\mathrm{p}_{\mathrm{r}}\right)$, a write dependency event placeClientOrderWriteDependency $\left(p_{r}, p_{c 2}\right)$ will be raised.

$\begin{array}{ll}\text { create rule } & \text { ruleName } \\ \text { event } & \text { failureRecoveryEvent } \\ \text { define } & \text { [viewName } \text { as }<\mathrm{OQL} \text { expression }>\text { ] } \\ \text { condition } & \text { [when condition] } \\ \text { action } & \text { recovery commands }\end{array}$

Fig. 5. Process interference rule structure

Similarly, a potential read dependent event is triggered if the failed process has at least one potentially read dependent process. The format of a read dependent event is $<$ readDependentProcessName>ReadDependency (failedProcess, rdProcess), such that readDependentProcessName is the name of the read dependent process, and rdProcess is the identifier of the read dependent process instance.

Define declares variables to support condition evaluation. A condition is a Boolean expression to determine the existence of process interference based on application semantics. Define and condition use the object model presented below as an interface to access the global execution history.

Action is a list of recovery commands. The command could invoke backward recovery of a process (deepCompensate), re-execution of a process, backward recovery of an operation, or re-execution of an operation.

Fig. 6 presents the global execution history object model as the interface to access the global execution history in the declare and condition clauses of a PIR. The object classes in the model include Process, Operation, and Delta. The methods of each class as well as the attributes and relationships among these classes provide the basis for retrieving the deltas associated with a specific process and/or operation, and also for identifying read and write dependent processes and operations. For example, the method getDeltas (className) is used to retrieve all the deltas of a given class name that are created by an operation or a process. Similarly getDeltas(className, attrName) further limits the returned deltas to be of a given attribute name identified by attrName.

Since the condition evaluation of a process interference rule contains a query over deltas generated by the normal execution and recovery activities of a process, a process has several types of operations to support querying over deltas. For example, getDeltasBeforeRecovery returns deltas that are created by the process before any recovery activity is performed, and getDeltasByRecovery returns deltas that are created by the recovery activities of a process. Class name (className) and attribute name (attrName) can be used as parameters for these methods to limit the returned deltas to those associated 


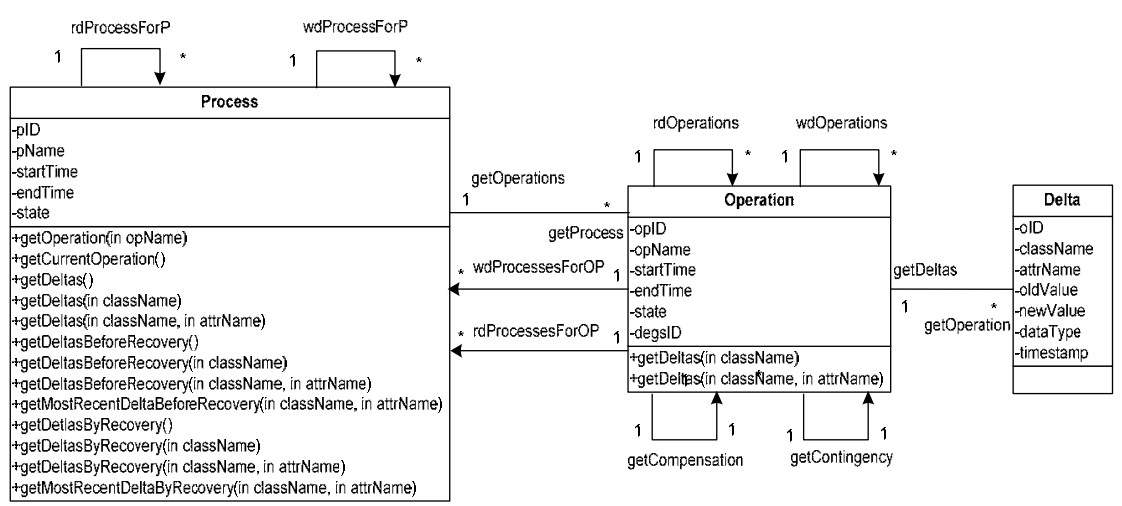

Fig. 6. Global Execution History Object Model

with a given class name and attribute name. getMostRecentDeltaBeforeRecovery(className, attrName) returns the most recent delta created by a process before the execution of any recovery activity, associated with a given class name and attribute name. Similarly, getMostRecentDeltaByRecovery(className, attrName) returns the most recent delta created by the recovery activities of a process.

\subsection{Example}

In Fig. 4, recall that the placeClientOrder process $\left(\mathrm{p}_{\mathrm{c} 2}\right)$ is write dependent on the process replenishlnventory $\left(p_{r}\right)$. As a result, $p_{\mathrm{c} 2}$ may process an order for a client based on an increase in inventory caused by $p_{r}$. If $p_{r}$ fails, however, after increasing the inventory for an item $x$ that is also accessed in $p_{c 2}$, then $p_{c 2}$ may also be affected if there will no longer be enough items in inventory for item $x$. As a result, $p_{c 2}$ may need to be backward recovered. Fig. 7 presents a PIR expressing the above application constraint.

The event is placeClientOrderWriteDependency(failedProcess, wdProcess). In this particular scenario, compensation of the process replenishlnventory $\left(p_{r}\right)$ raises the event placeClientOrderWriteDependency $\left(p_{r}, p_{c 2}\right)$ which matches the event defined in the rule.

The define clause creates bindings for inventory items (decreasedltems) that have been decreased by the recovery of failedProcess. The select statement finds deltas related to the quantity of an inventory item (getDeltasByRecovery("Inventoryltem", "quantity")) created by the recovery activities of failedProcess, finding items with decreased quantity by accumulating the changes on quantity for each item.

The condition has a when statement checking to determine if any decreased item (decltem) appears in the current client order. The evaluation is conducted by checking if any decltem is the same as the old of deltas associated with class Inventoryltem and attribute quantity created by wdProcess.

The action is to backward recover wdProcess from the current operation. The action will be performed if the when statement in the condition evaluates to true.

Similar rules can be developed to check user-defined conditions in the case of potential read dependencies. In this case, a PIR can be used to 1) determine if the failed process intersects with the potentially read dependent process on critical data 


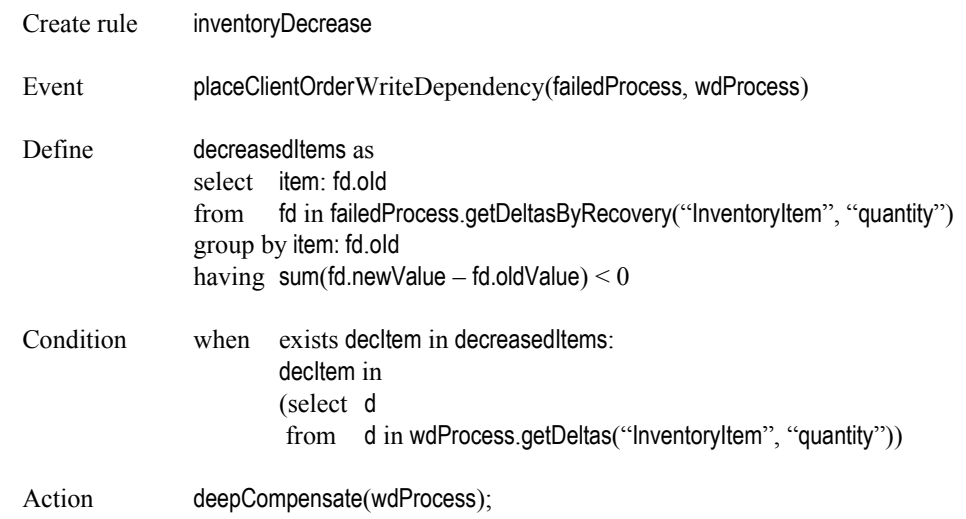

Fig. 7. PIR decreaseInventory for Process placeClientOrder

items by querying deltas on the process execution history, and 2) express applicationspecific conditions on the status of the critical data items after recovery of the failed process. If application constraints are violated, the read dependent process can invoke recovery procedures. Otherwise the read dependent process can resume execution. Further examples of PIRs for read and write dependencies can be found in [20].

\section{Conclusion and Future Directions}

This paper has presented a process dependency model together with the notion of process interference rules to resolve how the failure recovery of one process can potentially affect other concurrently executing processes in a distributed service composition environment. The resolution process is dependent on collecting information about data changes from delta-enabled grid services that are capable of forwarding these changes to a process history capture system [2]. We have implemented the PHCS [20, 21], which constructs a global execution history by merging deltas and process execution context. We have also fully implemented the PHCS object model to determine read and write dependencies and to invoke PIRs to determine if concurrent processes are affected by recovery of a failed process [20]. The research in [20] provides further details about simulation of the complete environment, with a performance evaluation of the implemented components. Our future work is focused on developing a distributed, peer-to-peer approach to the management and communication of deltas in resolving read and write dependencies, instead of forwarding deltas to a central process execution history component. We are also investigating the methodological issues associated with the use of process interference rules, as well as more dynamic approaches to the use of events and rules in the composition and recovery of distributed processes. 


\section{References}

1. Bhiri, S., O. Perrin, and C. Godart, "Ensuring required failure atomicity of composite Web Services," Proc. of the 14th int. conference on World Wide Web, 2005.

2. Blake, L., Design and implementation of Delta-Enabled Grid Services, MS Thesis, Deptment of Computer Science and Engineering, Arizona State Univ., (2005).

3. Web Services Transaction (WS-Transaction), http://www.ibm.com/developerworks/ library/ws-transpec/, 2002.

4. de By, R., W. Klas, and J. Veijalainen, Transaction management support for cooperative applications. 1998: Kluwer Academic Publishers.

5. Eder, J. and W. Liebhart, "The Workflow Activity Model WAMO," Proc. of the 3rd Int. Conference on Cooperative Information Systems (CoopIs), 1995.

6. Garcia-Molina, H. and K. Salem, "Sagas," Proc. of the ACM SIGMOD Int. Conference on Management of Data, 1987.

7. Jin, T. and S. Goschnick, "Utilizing web services in an Agent Based Transaction Model (ABT)," Proc. of the the 1st Int. Workshop on Web Services and Agent-based Engineering (WSABE' 2003) held in conjunction with the 2nd Int. Joint Conference on Autonomous Agents and Multi-Agent Systems, 2003. Melbourne, Australia.

8. Kamath, M. and K. Ramamritham, "Failure handling and coordinated execution of concurrent workflows," Proc. of the IEEE Int. Conference on Data Engineering, 1998.

9. Limthanmaphon, B. and Y. Zhang, "Web Service composition transaction management," Proc. of the 15th Australasian database conference, 2004. Dunedin, New Zealand: Australian Computer Society, Inc.

10. Lin, F., Chang, H., "B2B E-commerce and enterprise Integration: the development and evaluation of exception handling mechanisms for order fulfillment process based on BPEL4WS," Proc. of the 7th IEEE Int. Conference on Electronic commerce, 2005.

11. Lomet, D., Z. Vagena, and R. Barga, "Recovery from "bad" user transactions," Proc. of the ACM SIGMOD Int. Conference on Management of Data, 2006.

12. Mikalsen, T., S. Tai, and I. Rouvellou, "Transactional attitudes: reliable composition of autonomous Web Services," Proc. of the Workshop on Dependable Middleware-based Systems (WDMS), part of the Int. Conference on Dependable Systems and Networks (DSN), 2002.

13. Peri, R.S., Compilation of the Integration Rule Language, MS Report, Department of Computer Science and Engineering, Arizona State University, (2002).

14. Shi, Y., L. Zhang, and B. Shi, "Exception handling of workflow for Web Services," Proc. of the 4th Int. Conference on Computer and Information Technology, 2004.

15. Singh, M.P. and M.N. Huhns, Service-Oriented computing. 2005: Wiley.

16. Tartanoglu, F., et al., "Dependability in the Web Services architecture," Proc. of the Architecting Dependable Systems, LNCS 2677, 2003.

17. Urban, S.D., et al., "The IRules Project: using active rules for the integration of distributed software components," Proc. of the 9th IFIP 2.6 Working Conference on Database Semantics: Semantic Issues in E-Commerce Systems, 2001.

18. Wachter, H. and A. Reuter, "The ConTract model," in Database transaction models for advanced applications, A. Elmagarmid, Editor. 1992.

19. Worah, D. and A. Sheth, "Transactions in transactional workflows," in Advanced transaction models and architectures, S. Jajodia and L. Kershberg, Editors. 1997, Springer.

20. Xiao, Y., Using deltas to support semantic correctness of concurrent process execution, Ph.D Dissertation, Department of Computer Science and Engineering, Arizona State Univ., (2006). 
21. Xiao, Y., S.D. Urban, and S.W. Dietrich, "A process history capture system for analysis of data dependencies in concurrent process execution," Proc. of the 2nd Int. Workshop on Data Engineering Issues in E-Commerce and Services, 2006.

22. Xiao, Y., S.D. Urban, and N. Liao, "The DeltaGrid abstract execution model: service composition and process interference handling," Proc. of the 25th Int. Conference on Conceptual Modeling, 2006.

23. Zeng, L., et al., "Policy-driven exception-management for composite Web Services," Proc. of the 7th IEEE Int. Conference on E-Commerce Technology, 2005. 